Submitted to Astrophysical Journal

\title{
ON THE ONGOING MULTIPLE BLOWOUT IN NGC 604
}

\author{
Guillermo Tenorio-Tagle \\ INAOE, Apartado Postal 51, Puebla, Pue. MEXICO \\ Casiana Muñoz-Tuñón \\ Instituto de Astrofísica de Canarias, 38200 La Laguna, Tenerife, SPAIN \\ Enrique Pérez \\ Instituto de Astrofísica de Andalucía (CSIC), Aptdo. 3004, 18080 Granada, SPAIN \\ Jesús Maíz-Apellániz \\ Space Telescope Science Institute, 3700 San Martin Drive, 21218 Baltimore, USA \\ Laboratorio de Astrofísica Espacial y Física Fundamental-INTA, Apartado Postal 50727, 28080 \\ Madrid, SPAIN \\ Gustavo Medina-Tanco \\ Instituto Astronômico e Geofísico, University of São Paulo, BRAZIL
}

\begin{abstract}
Several facts regarding the structure of NGC 604 are examined here. The three main cavities, produced by the mechanical energy from massive stars which in NGC 604 are spread over a volume of $10^{6} \mathrm{pc}^{3}$, are shown here to be undergoing blowout into the halo of M33. High resolution long slit spectroscopy is used to track the impact from massive stars while HST archive data is used to display the asymmetry of the nebula.

NGC 604 is found to be a collection of photoionized filaments and sections of shells in direct contact with the thermalized matter ejected by massive stars. The multiple blowout events presently drain the energy injected by massive stars and thus the densest photoionized gas is found almost at rest and is expected to suffer a slow evolution.
\end{abstract}

Subject headings: ISM: kinematics and dynamics - H II regions - NGC 604 


\section{INTRODUCTION}

Upon the formation of massive stellar clusters, the energy input from massive stars leads to a rapid structuring of the surrounding medium. This was originally thought to be due to the high pressure acquired by the constant density photoionized gas which, upon the formation of the H II region, would then expand with a speed smaller than the sound speed $\left(c_{H I I} \sim 10 \mathrm{~km} \mathrm{~s}^{-1}\right)$ onto the surrounding neutral gas to acquire a radius

$$
R_{H I I}(t)=R_{S t}\left(1+7 / 4\left(c_{H I I} t / R_{S t}\right)\right)^{4 / 7}
$$

where $R_{S t}$ is the Strömgren radius (Spitzer 1968; Osterbrock 1989). Much larger speeds were envisaged in "champagne flows" as a result of the pressure gradient between the photoionized parent cloud and the photoionized inter-cloud medium, suddenly established as the ionization front (either $\mathrm{D}$ or $\mathrm{R}$ type) overruns the parent cloud edge (Tenorio-Tagle 1979). In this case the ionization front rushes to infinity and strong isothermal shocks develop within the photoionized region. The shocks are driven by the ionized cloud matter that supersonically streams into the intercloud medium in an attempt to lift and equalize the pressure between the two regions. The champagne phase would then last until the pressure gradient is smoothed out; fact that is accomplished only once the whole ionized cloud is dispersed.

A completely new paradigm resulted from taking into consideration the mechanical energy produced by massive stars and the evolution of superbubbles. In this case three concentric distinct regions were shown to develop around the exciting source (Castor et al. 1977): The central region (a) contains the hypersonic wind from the massive $\operatorname{star}(\mathrm{s})$. At a certain radius, $R_{a}$, this is abruptely thermalized at a reverse shock. Given the speed of the wind, the high temperature $\left(T=1.4 \times 10^{7}\right.$ $\left.\left(v_{\text {wind }} / 1000 \mathrm{~km} \mathrm{~s}^{-1}\right)^{2} \mathrm{~K} \sim 10^{6}-10^{7} \mathrm{~K}\right)$ and high pressure acquired upon thermalization, allow the shocked wind to fill a large volume (region $b$ ), while driving a strong shock into the surrounding gas. The latter shock then sweeps and accelerates the surrounding ISM into a dense expanding shell (region $c$ ), that grows as a function of time as (Castor et al. 1977; Weaver et al. 1977)

$$
R_{s}(t)=0.76(\dot{E} / \rho)^{1 / 5} t^{3 / 5}
$$

where $\dot{E}$ is the mechanical energy input rate and $\rho$ the mass density of the background medium. Castor et al. (1977) also showed that, given the number of recombinations within the expanding shell and its rapid growth, the ionization front became trapped within the shell early in the evolution of the system. This was shown to happen despite the fact that most of the volume is filled with the hot thermalized wind, which is a transparent medium to the ionizing photon flux due to its high temperature. Consequently, the $\mathrm{H}$ II region gas in this model was shown to be rapidly confined to a narrow 2D structure within the expanding shell.

Other models, accounting only for the mechanical energy deposited by OB associations and starbursts (Tenorio-Tagle \& Bodenheimer 1988), have clearly shown how the shells of swept up matter can easily be disrupted upon crossing a large density gradient. This phenomenon, commonly referred to as blowout, occurs as a shell suffers a sudden acceleration, fact that drives it Rayleigh- 
Taylor unstable and causes its rapid fragmentation. The event then leads to the venting of the hot superbubble interior onto the low density gas, where a new shell of halo swept up matter will develop. More recent studies have also shown that blowout also leads to the escape of the $u v$ photons, which otherwise would be trapped within the decelerating shell. The escape of ionizing radiation has been shown to lead to the development of an extended, low surface brightness conical H II region ahead of the superbubble leading shock (Tenorio-Tagle et al. 1999).

Here we center our attention on the nearest northern hemisphere giant H II region NGC 604. The giant nebula is the largest in the neighbouring spiral galaxy M33 (at a distance of $840 \mathrm{kpc}$; Freedman et al. 1991), and is located in one of the spiral arms, $2.4 \mathrm{kpc}$ from the galaxy centre. Looking at NGC 604, it is quite evident that it is not a spherical nebula. It presents a large number of shells, filaments or arches of a wide variety of lengths and intensities. Its general appearance is, however, very similar to other giant $\mathrm{H}$ II regions, in particular to the 30 Doradus nebula. The large number of shells and networks of shells in 30 Doradus have been identified spectroscopically by Chu \& Kennicutt (1994). Such a structure, i.e. expanding shells within shells, imply a very inhomogeneous rapidly structured, or pre-structured, parent cloud density distribution. An endless number of tunnels, or cavities, across which the thermalized winds from the massive stars could freely flow causing their expansion and further growth, as to in projection allow for the appearance of a network of nested shells. In such a case, the thermalized ejecta, the hot coronal gas, is to be in direct contact with the HiI region gas, and the latter would be confined to the narrow denser walls of the network of tunnels or to the sections of the expanding shells, presenting thus a three dimensional superposition of multiple 2D structures.

Here we analize the ionization structure, and the velocity field of NGC 604, with the aim of unveiling its true morphology and possible evolution of the nebula. In section 2 we analyse the two data sets used in this work, and in section 3 we present a discussion of the results and our conclusions.

\section{Analysis and results}

\section{1. $\quad H S T$ imaging}

We have retrieved from the $H S T$ archives narrow band images in the emission line filters

of $\mathrm{H} \alpha(\mathrm{F} 656 \mathrm{~N}),[\mathrm{O} \mathrm{III}] \lambda 5007(\mathrm{~F} 502 \mathrm{~N})$, and [S II] $\lambda 6717+6731(\mathrm{~F} 673 \mathrm{~N})$. Each final image is the combination of two $1100 \mathrm{~s}$ exposures, using the STSDAS tasks crrej and wcombine. Figure 1 shows the image ratios corresponding to $[\mathrm{O}$ III $] / \mathrm{H} \alpha$ and $[\mathrm{S} \mathrm{II}] / \mathrm{H} \alpha$.

A cursory look at these two image ratios shows that, as expected, they trace distinct different regions of the nebula. The $[\mathrm{S} \mathrm{II}] / \mathrm{H} \alpha$ ratio traces the low excitation regions and is not affected by extinction. The $[\mathrm{O} \mathrm{III}] / \mathrm{H} \alpha$ ratio maps the high excitation regions, but it can be affected by extinction, so that a high value always indicates high excitation, but a low value can be a combination 
of both low excitation and/or high extinction. This technique of using narrow band emission line imaging ratios has been used successfully in the literature to study the ionization structure and radiation field in different types of photoionized sources, such as Seyfert and Starburst galaxies, where ionization cones and other collimated structures have been thus uncovered (Tadhunter \& Tsvetanov 1989; Pérez et al. 1989).

The high excitation $\mathrm{O}^{++}$region is maintained by radiation with at least $35 \mathrm{eV}$, while the low excitation $\mathrm{S}^{+}$zone requires only of photons more energetic than $10 \mathrm{eV}$. Thus, in an idealized constant density H II region, with a source of $u v$ photons arising from the center of the nebula, the $\mathrm{S}^{+}$zone should be concentric and surrounding the $\mathrm{O}^{++}$zone. This is clearly not the case in NGC 604 (see Figure 1). Instead, although the high excitation region is largely confined by the outer low excitation region, there exists a large, almost straight dividing line that spans across $\sim$ 200 pc (50 arcsec) almost through the center of the nebula (offset by some 10 arsec), running from the extreme north to the southern edge. The sharp boundary between the two emitting volumes (hereafter referred to as the "ridge"), has no explanation within the standard case. Note however, that (unlike R136 in 30 Doradus) the ionizing cluster in NGC 604 is not centrally concentrated, but rather the 186 massive $\mathrm{O}$ stars visible in the HST images are here distributed over a projected area of about $10000 \mathrm{pc}^{2}$ (Hunter et al 1995; Terlevich et al 1996; see figure 2 in González Delgado \& Pérez 1999). Furthermore, from a recent radio continuum and $\mathrm{H} \alpha$ study, Churchwell \& Goss (1999) conclude that the main emission centres (their knots A-F) are also photoionized from the inside by heavily obscured massive stars, and an average of five or more $\mathrm{O}$ stars are required to produce the ionization of each of the condensations. These come to enhance the number of massive O stars to more than 220, scattered over the projected central $10000 \mathrm{pc}^{2}$ area. Also from this work an extinction map was obtained with values ranging from 0.3 to 0.5 magnitudes, thus implying that we see most of the nebula without obscuration (see also Díaz et al. 1987). Another recent analysis (Maíz-Apellániz et al. 2000) finds a discrepancy between the extinction measured from the Balmer decrement and the one found by Chuchwell \& Goss (1999), suggesting that a large fraction of the obscuring dust is mixed with or located close to the gas. This together with the CO detection (Viallefond \& Goss 1986) implies the existense of a molecular cloud behind the photoionized nebula NGC 604.

The length of the ridge between the two different excitation regions demands the existence of a sharp discontinuity across which distinct physical conditions operate and is most likely caused by the existence of a molecular wall (Wilson \& Scoville 1992, Maíz-Apellániz et al. 2000). The ridge, in deep $\mathrm{H} \alpha$ images seems to result from the chance alignment of the three main shells, or superbubbles, whose sharp and elongated edges coincide to give the appearance of a long ridge (see Figures 1 and 2).

Further information regarding the structure of the region can be derived from the kinematics of the ionized gas. In what follows we analyse spectra taken at the WHT (4.2m) at the Observatorio del Roque de los Muchachos using the double arm ISIS spectrograph. 


\subsection{The peak intensity features}

A 1 arcsec wide slit was placed east-west at ten different locations stepped by 2 or 3 arcsec across the nebula (the step between slit positions \#6 and \#7 is 2 arcsec). The sampling along the spectral and spatial direction in the slit is $0.37 \AA$ pixel $^{-1}$ and $0.33 \operatorname{arcsec}_{\text {pixel }}{ }^{-1}$, respectively. The precise location of the slits is shown in Figure 2 and a detailed description of the observational set-up and data reduction can be found in Mas-Hesse et al (1994), Maíz-Apellániz (1999), and Maíz-Apellániz et al (1999). Figures 3a to 3j display the measured velocity and flux of the $H \alpha$ emision line along each of the slits crossing the nebula. The measurements have been made by fitting a single Gaussian component to the observed $\mathrm{H} \alpha$ emission line, except where a good fit to the line required two or sometimes three components. The velocity field $\left(\mathrm{km} \mathrm{s}^{-1}\right)$ is derived from the center of the Gaussian fits, referred to the $\mathrm{H} \alpha$ rest wavelength $\left(\lambda_{0}=6562.58 \AA\right)$. The line intensity scale (in arbitrary units) gives an indication of the density, or the column density at the observed locations. The median velocity is $256 \pm 9 \mathrm{~km} \mathrm{~s}^{-1}$.

The long ridge that divides the east and west sides of the nebula is evident in all the slits. The feature presents a maximum intensity at pixel $\sim 300$ on all slits. The central velocity at such local maxima is very uniform with values of $-250 \mathrm{~km} \mathrm{~s}^{-1}$ to $-260 \mathrm{~km} \mathrm{~s}^{-1}$ across the ten slits. These are very similar to the galaxy local reference frame velocity. The sharp ridge across the nebula is most probably a high density wall with a very steep drop in intensity towards the east, noticeable on all slits. Much further away (around location 370) one finds the Big Loop. This is easily identified in Figure 2 across slits 1 to 5 (see insert sketch).

Further out on slit 1, at positions 430 and 445, the two small local maxima correspond to two small H II regions (Figure 2) and both also present a velocity of $-260 \mathrm{~km} \mathrm{~s}^{-1}$. The two point-like $\mathrm{H}$ II regions have been used as reference for the positioning of the slits.

Churchwell \& Goss (1999) have identified six radio continuum maxima and labelled them as A-F. In what follows we shall keep their nomenclature (see Figure 2). The brightest zones of the nebula are crossed by slits 6 to 10. The sharpest peak is knot A easily found on slit 8 at pixel 300 . Its spatial extent, measured as the apparent width at half intensity, is about 5 pixels, or 1.8 arcsec, equivalent to $7.9 \mathrm{pc}$, if one assumes a distance scale of $4.1 \mathrm{pc} \operatorname{arcsec}^{-1}$. Knots $\mathrm{A}$ and $\mathrm{B}$ (on slits 8, 9 and 10) lie on top of a high density wall and in fact between them conform the most evident southern part of the long ridge.

The other main emitting knots are also clear in our spectra. Knot D is very elongated and most evident on slits 4 to 7 . It is brighter on slit 6 (with $10^{4}$ counts at position 245) and of comparable brightness on slit 7 (9000 counts at 250). The intensity of this features slowly fades through slits 5 (5500 counts at 245), 4 (3200 counts at 240), 3 (2300 counts at 245) and 2 (1700 counts at 240), and it is absent on slit 1. A deep inspection of the image shows that in fact, the feature ends up running almost parallel to slit 2 (from position 250 to 280). Therefore, although along the slit direction, knot $\mathrm{D}$ is relatively narrow ( $\sim 15$ pixels wide at half intensity on slit 7 ), it extends continously from the north-west northwards and then to the east following a concave curvature. The emission 
increases once again as one moves towards the south across slits 3-7 (positions 280-290). Towards the south the feature acquires large intensity values, similar to those found along the extension of knot $\mathrm{D}$ towards the north. If one follows this roundish shape moving onto slit 8 one finds the next maximum in intensity (knot $\mathrm{C}$ ). In our spectra this splits into two distinct knots, each of them with typical sizes of 10 pixels ( $~ 3.7$ arcsec width at half intensity), equivalent to $15.2 \mathrm{pc}$.

With the exception of knots $\mathrm{A}$ and $\mathrm{B}$, the intensity maxima described above clearly define a central zone with a much lower $\mathrm{H} \alpha$ intensity. There, the flux measured in between maxima reaches the lowest possible values (as on slits 4 and 5). There seems to be an empty hole, the boundary of which is well delineated by some of the main emitting knots and by the slightly curved central section of the ridge on the east side. We have then identified an elliptical shape cavity with a diameter $\sim 40$ pixels $(\sim 15 \operatorname{arcsec} \times 11.25 \operatorname{arcsec}$ or $61.5 \mathrm{pc} \times 46.2 \mathrm{pc})$.

Within the central hole we identify a small but extended zone (the "intrusion"), which extends (E-W) from pixel 300 to 265 along slit 6, i.e. some 15 pixels $(22.75$ pc) while reaching a maximum flux of 2500 units.

\subsection{The velocity field}

The two reference H II regions (at pixels 435 and 445 on slit 1), both ends of every slit and all obvious intensity maxima traversed by the slits (with the exception of the intrusion in the main cavity) show the same velocity, $-255 \pm 10 \mathrm{~km} \mathrm{~s}^{-1}$. This value, has then been assumed as the galaxy reference frame.

The velocity curves also display a different trend between the [S II $]$ and the [O III] dominated regions. There is no zone within the east side of the $[\mathrm{S} \mathrm{II}]$ region that shows line splitting. All slit positions within this [S II] dominated region, regardless of the $\mathrm{H} \alpha$ intensity, are well fitted with a single Gaussian with peak velocities about -260 to $-280 \mathrm{~km} \mathrm{~s}^{-1}$, all within $10 \mathrm{~km} \mathrm{~s}^{-1}$ from the rest-frame velocity of M33.

One can therefore conclude that there are no signs of this region being affected by the mechanical energy from the massive stars. The [S II] zone presents in fact evidence of a slow radial expansion with respect to the M33 reference frame velocity. Note also that not even the largest loop of NGC 604, apparent at all wavelengths at the outer edge of the nebula (at the extreme North-East edge, across slits 1-6), presents any sign of line splitting and thus we regard it as an old structure at the edge of a molecular cloud. The exact opposite is true within the [O III] dominated zone. There the velocity field, through large line splitting, evidences the mechanical power from massive stars, capable of displacing the ISM as it generates giant cavities.

Apart from the obvious main cavity, there are also several smaller less obvious holes, the borders of which are also traced in the HST $\mathrm{H} \alpha$ image. The largest ones (cavities 2 and 3, see 
Figure 2) are to the south and north, immediately adjacent to the rim of the central hole, and are bound on their eastern side by the ridge. The two of them fade away in opposite directions, cavity 2 appears open and fainter towards the south (see slits 8-10 at pixels 260 to 300), while cavity 3 towards the north (see slits 1-3 at pixels 280 to 300). Other even smaller and fainter features are even less apparent and it is a difficult task to describe them with the same accuracy.

Line splitting has been detected in all three cavities. The largest inferred expansions from the spectroscopic data are $\sim 100 \mathrm{~km} \mathrm{~s}^{-1}$ with respect to the galaxy rest frame. In the regions of line splitting, the two line components are displaced from the galaxy rest frame velocity. The redshifted component acquires in all cases a maximum value $\sim-240 \mathrm{~km} \mathrm{~s}^{-1}$. It is also the redshifted component, with exception of that arising from the intrusion feature in the main cavity, the one that invariably presents the largest intensity. On the other hand, the blue shifted components reach values of $-370 \mathrm{~km} \mathrm{~s}^{-1}$, implying expansion speeds of up to $100 \mathrm{~km} \mathrm{~s}^{-1}$ with respect to the galaxy rest frame. The blue shifted components present also in all cases a low intensity flux, always below 100 counts (see Figure 3), except in the intrusion feature seen in projection within the main cavity. The "intrusion" thus appears as a dense feature (intensity $\sim 2000$ counts at pixel 270, on slit 6), blueshifted some $20 \mathrm{~km} \mathrm{~s}^{-1}$ with respect to the galaxy rest frame velocity. At the same position the redshifted counterpart reaches speeds $\sim-220 \mathrm{~km} \mathrm{~s}^{-1}$, very similar to those detected at the same pixel location on the neighbouring slits 5 and 7 . There are also some regions, very few, which display more than two components. These are all found within the main cavity. A precise modeling of these is difficult to do, particularly when the measured parameters are affected by large uncertainties. All one can assure is that more than two Gaussian components are required to reproduce the emission line profile. We must centainly be intercepting remnant shells or low intensity filaments along the line of sight, stressing the idea of depth or of a 3D structure of the nebula.

Note however that the velocity centroids of the splitted lines do not conform, in any of the studied cases, to complete velocity ellipsoids, as those expected from an ionized shell expanding into a uniform density medium; i.e., an ellipsoid showing maximum speed of approach and recession when looking across the centre of the cavities, steadily merging with the galaxy rest-frame velocity as one approaches the borders of the expanding structure (see, e.g. Maíz-Apellániz et al. 1999). The data instead shows distinctly open structures. Line splitting is clearly found even when looking over some of the borders, or rims, of the most obvious cavities (see slits 2 and 3 ).

Figure 4 maps the velocity centroids of the two main line splitting components across the north-south direction and through the centres of cavities 1,2 and 3. Figure 4a shows the range of velocities detected at pixel 270 . Figures $4 \mathrm{~b}$ and $\mathrm{c}$, show the trend of the blue and the red shifted components in cavities 2 (pixel 285) and 3 (pixel 290), respectively. In the three cases the velocity ellipsoids expected from shell-like structures expanding onto the parent cloud are not recovered. The line spliting shown in all of them leads to widely separated velocity features with a $\Delta v$ in the range of $60-70 \mathrm{~km} \mathrm{~s}^{-1}$. Following the slits across the cavities, i.e., along the east-west direction (see Figure 3), one can see that the line splitting is confined by the borders of cavities. Although 
slit 2 at position $\sim 280$ presents evidence of a large line splitting right at the high intensity border between cavities 1 and 3 . This fact indicates that the blue shifted outflows from these cavities have already managed to surpase the dimensions of the cavities that originally confined them. The trend detected in the three main cavities of NGC 604 thus resembles the trace left by the cones that channel superwinds out of starbursts galaxies, as in NGC 253, and M82 (see Heckman et al. 1990). NGC 604 being a much smaller and weaker source, represents a small-scale version of a superwind event. However, it is a clear example of the venting of the hot superbubble content into the halo of M33, as well as of the streaming or leakage of $u v$ radiation into the halo of M33.

Positions with line splitting associated to cavities 2 and 3 are also found at locations far from the main extended region of star formation. The line splitting at these positions appears to coincide with radial gaseous filamentary regions at the edge of the emitting zone. The structures thus resemble the tracing of conical outlets through which the hot gas, which otherwise could be used to power the expansion of bubble-like structures, freely expands away from NGC 604, most probably into the low density halo of M33.

Typical of the splitted lines, is their low intensity fast blue shifted expanding components, much faster than those detected in the redshifted counterparts. Also, note that the blue shifted components present in all cases, but in the intrusion, a much lower intensity, indicative of a recent sudden burst into a lower density medium. Both of these arguments together with the fact that the blue shifted velocity component is also seen on top of the projected boundaries of the cavities (as in slits 2 and 3), support the evidence of an ongoing blowout. In this scenario, the intrusion feature corresponds to the densest remaining part of a shell that has undergone blowout in our general direction. It seems also relevant to point at the elongated rim of cavity 3 which may have developed during blowout, and that spans across to conform the northern half length of the long ridge.

\section{Discussion and Conclusions}

Central to the study of HII regions is to define the way they evolve and for this an accurate description of their morphology is a key issue to confront with theoretical models. NGC 604 is excited by a widely spread cluster of some 200 massive stars, and these have produced two nonconcentric different excitation zones distinctly different also from a kinematical point of view. Our kinematical data provides also strong evidence of an ongoing multiple blowout of the photoionized nebula into the halo of M33.

The [O III] dominated section of NGC 604 presents a corrugated multiple shell appearance, presently bursting along several directions. This is most evident across bubbles 1, 2, and 3 and along the filamentary channels detected at large radii, both along the north and southern edges of the nebula. Note that bubbles 2 and 3 spread out far from the extended cluster of massive stars. Furthermore, in all these cases line splitting consistently shows the densest (high intensity) gas only 
slightly redshifted with respect to the galaxy reference frame, and thus it is expected to evolve very slowly during the life time of the exciting stars. On the other hand, the low intensity blueshifted counterpart is clearly affected by a rapid evolution, as expected from the surface of superbubbles bursting towards us at high speeds into the halo of M33.

The eastern [S II] section of NGC 604 presents a completely different structure that seems kinematically unaffected by the winds and supernovae from the extended exciting cluster. This conclusion arises from the absence of line splitting and the lack of bubble-like structures. The [S II] region in fact gives the impresion of a large photoionized section of the surface of an extended cloud expanding away from us with a velocity smaller than $10 \mathrm{~km} \mathrm{~s}^{-1}$.

Many models have been used to explain diffuse ionized nebulae (see section 1). However, to account for the observed facts above, a particular scenario is required. This involves a pillar morphology of the parent molecular cloud, where massive star formation must have been triggered at one end and deep along its surface, perhaps by a major disturbance such as the M33 spiral density wave. The star formation event covers in projection an area of some $10500 \mathrm{pc}^{2}$ and must probably occupy a volume $\sim 10^{6} \mathrm{pc}^{3}$. We envisage star formation as to have occured in many places deep enough inside the cloud, and as closely spaced together, as to allow the mechanical energy from massive stars to build up a network of twisted tunnels, filled with the thermalized ejecta; in a similar manner to that envisaged by Cox \& Smith (1974) for OB associations in the galaxy. The tunnels will then traverse the tip of the giant molecular cloud and become broader, particularly in the outward direction; i.e. away from the symmetry axis or densest part of the cloud, in the places where more energy is deposited per unit time, or where the local surrounding cloud density is the lowest. In some of these places the energy from the "super-tunnels" will even burst out of the cloud, releasing the hot coronal gas into the low density surrounding medium.

Looking at the cloud along its symmetry axis, the enhanced diameter sections of the cylindrical tunnels would appear like the borders, or rims, of individual "shells", and the collection of protuberances along the surface of a tunnel could cause the apearance of networks of nested shells.

NGC 604 has three large cavities evident in the HST H $\alpha$ image. The rims of these conform the broad borders of cavity 1 and of the neighbouring more distorted "bubbles" 2 and 3 . The alignment of the three borders, jointly compose the dense ridge that separates the high from the low excitation regions of the nebula. Note that the end of cavity 3 is the most distorted of them all, and extends for some distance away from the center of star formation. The walls of the three cavities must extend deep into the cloud to inhibit in this way an important leakage of hard uv radiation into the $[\mathrm{S}$ II] main emission section of the nebula.

From the kinematic data we know that the three main bubble structures have burst into the halo of M33, as evidenced by the line splitting across their projected cross-section. This confirms the detection of the main bubbles through a completely different observational approach using Fabry-Perot data (see Muñoz-Tuñón et al. 1996). The cavities, or tunnels, may have also burst in other directions, leading to the giant faint loop structures, and filamentary structures seen at 
large projected distances from the star forming zone. These regions being far from the collection of exciting stars present thus a low ionization parameter and are most evident in $[\mathrm{S} \mathrm{II}] / \mathrm{H} \alpha$.

Clearly a detailed model of NGC 604 should strongly depart from the idealized one dimensional spherical symmetry, while allowing photoionization and the stellar wind energy input to jointly work on the shaping of the parent cloud. The above thus implies that:

a) In NGC 604 the formation of massive stars has taken place within a large volume ( $10^{6} \mathrm{pc}^{3}$ ), and the mechanical energy from these has rapidly structured the parent cloud into a collection of interconected bubbles and tunnels filled with the thermalized matter ejected in winds and eventually SN explosions.

b) Consequently, the $\mathrm{H}$ II region is not filled with photoionized gas and thus it cannot possibly expand as originally thought $\mathrm{H}$ II regions would do (see section 1). The matter surrounding massive stars has rapidly been confined to the borders or walls of the multiple bubbles and tunnels, and there, exposed to the $u v$ radiation causes the multiple shell or filamentary appearance characteristic of giant H II regions.

c) The hot coronal gas that initially drives the expansion of the network of bubbles and tunnels moves rapidly across the organized network to remain in direct contact, and thus at the same pressure, as the high excitation photoionized section of the nebula.

d) The evidence of blowout into the halo of M33, also implies that the expansion velocity of tunnels and shells should rapidly plummet as the driving energy escapes into the halo of the galaxy. In this case, the general structure is expected to suffer little changes during the remaining life-time of the exciting stars.

e) Blowout should allow the hot coronal medium to engulf the whole cloud and to establish a new pressure balance along the extended cloud surface.

f) Far away photoionized sections of the cloud would inevitably suffer their disruption through champagne flows, although details of such flows once the cloud surface is also permeated by the hot coronal medium, remain to be worked out.

g) As a consequence of blowout, an important and increasingly larger amount of $u v$ photons escape the nebula. This should largely contribute to the ionization of the extended halo of M33.

Acknowledgements We gratefully acknowledge the hospitality of the Guillermo Haro program for advanced astrophysical research (workshop 1999) which largely promoted our collaboration in this project. We are also grateful to the CONACYT (México) - DGES (Spain) proyect 97-0158, and CSIC-CONACYT project 99MX0021 as well as the CONACYT project 28501-E (México), and FAPESP/CNPq (Brazil). We acknowledge the hospitality of the Instituto de Astrofísica de Andalucía (CSIC, Granada, Spain) and the Institute of Astronomy (Cambridge, UK). Our thanks also to David Carter for his help with gathering of the long-slit data within the GEFE collaboration and to Miguel Mas-Hesse for his making sure we could count on very well calibrated data. 


\section{REFERENCES}

Castor J., McCray R., \& Weaver R., 1975, ApJ, 200, L107

Chu, Y.-H., \& Kennicutt, R.C.Jr., 1994, ApJ, 425, 720

Churchwell, E. \& Goss, W. M., 1999, ApJ 514, 188

Cox, d. p. \& Smith B. W., 1974, ApJ 189, 105

Díaz, Á.I., Terlevich, E., Pagel, B.E.J., Vílchez, J.M., Edmunds, M.G., 1987, MNRAS, 226, 19

Freedman, W.L., Wilson, C.D. \& Madore, B.F., 1991, ApJ, 372, 455

González-Delgado, R.M., et al., 1994, ApJ, 437, 239

González-Delgado, R.M., Pérez, E., 1999, MNRAS, submitted

Heckman, T.M., Armus, L. \& Miley, G.K., 1990, ApJS, 74, 833.

Hunter, D.A., Baum, W.A., O’Neil, E.J.Jr., Lynds, R., 1996, ApJ 456, 174

Maíz-Apellániz, J., 1999, Ph.D. Thesis, Univ. Complutense, Madrid.

Maíz-Apellániz, J., Muñoz-Tuñón, C., Tenorio-Tagle, G. \& Mas-Hesse, M., 1999, A\&A, 343, 64.

Maíz-Apellániz, J., Pérez, E. \& Mas-Hesse, M., 2000, in preparation.

Mas-Hesse, M., Muñoz-Tuñón, C., Vílchez, J. M., Castañeda, H. O. \& Carter, D., 1994, in Violent Star Formation: From 30 Doradus to QSOs, ed. Tenorio-Tagle, CUP, p. 125.

Muñoz-Tuñón, C., Tenorio-Tagle, G., Castañeda, H. O. \& Terlevich, R. 1996, AJ, 112, 1636

Osterbrock, D. E., 1989, in Astrophysics of Gaseous Nebulae and Active Galactic Nuclei, University Science Books, Mill Valley.

Pérez, E., González-Delgado, R.M., Tadhunter, C.N., Tsvetanov, Z., 1989, MNRAS 241, 31P

Spitzer L.: 1968: in Diffuse Matter in Space, Interscience Publishers. John Wiley \& Sons Inc. New York, London, Sydney, Toronto.

Tadhunter, C.N., \& Tsvetanov, Z., 1989, Nature, 341, 422

Tenorio-Tagle, G., 1979, A\&A, 71, 59

Tenorio-Tagle, G. \& Bodenheimer, P., 1988, ARA\&A, 26, 145

Tenorio-Tagle, G., Silich, S., Kunth, D., Terlevich, E., Terlevich, R., 1999, MNRAS, 309, 332

Terlevich, E., Díaz, Á.I., Terlevich, R.J., González-Delgado, R.M., Pérez, E., García Vargas, M.L., 1996, MNRAS 279, 1219

Viallefond, F., \& Goss, W.M., 1986, A\&A, 154, 357

Weaver R., McCray, R., Castor, J., Shapiro, P. \& Moore, R., 1977 ApJ, 218, 377

Wilson, C. D. \& Scoville, N., 1992, ApJ, 385, 512 


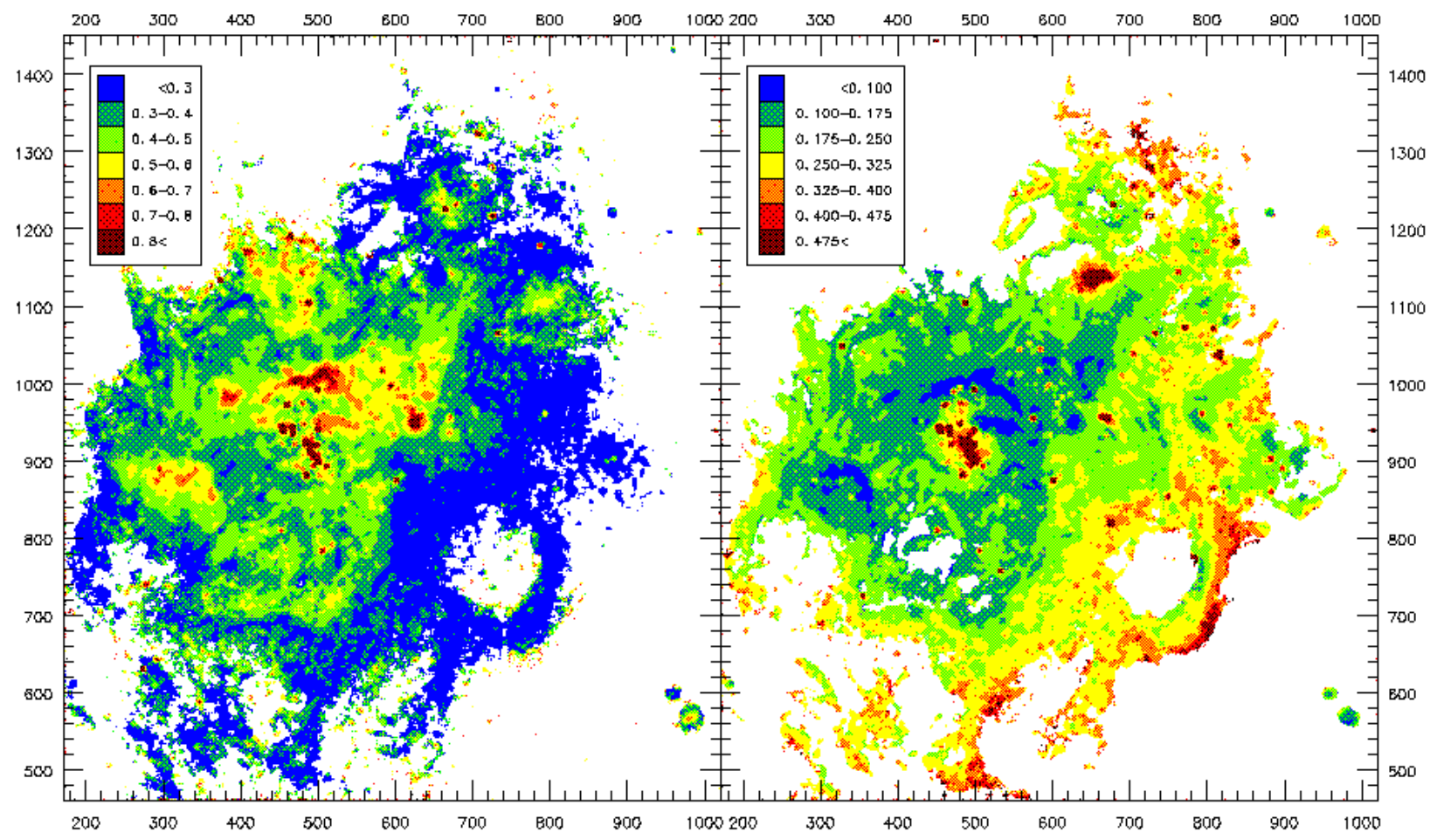

Fig. 1.- $[\mathrm{O} \mathrm{III}] / \mathrm{H} \alpha$ (left) and $[\mathrm{S} \mathrm{II}] / \mathrm{H} \alpha$ (right) excitation maps derived from the HST WFPC2 images of NGC 604. The scale used for each of the ratios is indicated in the legend. Note that for $[\mathrm{O} \mathrm{III}] / \mathrm{H} \alpha$ high values correspond to high excitation while for $[\mathrm{S} \mathrm{II}] / \mathrm{H} \alpha$ high values correspond to low excitation. The axes are labelled in WF pixels, with 1 pixel $\approx 0 ! \prime 1$. North points towards the lower left corner, forming an angle of 27.72 with the vertical. 


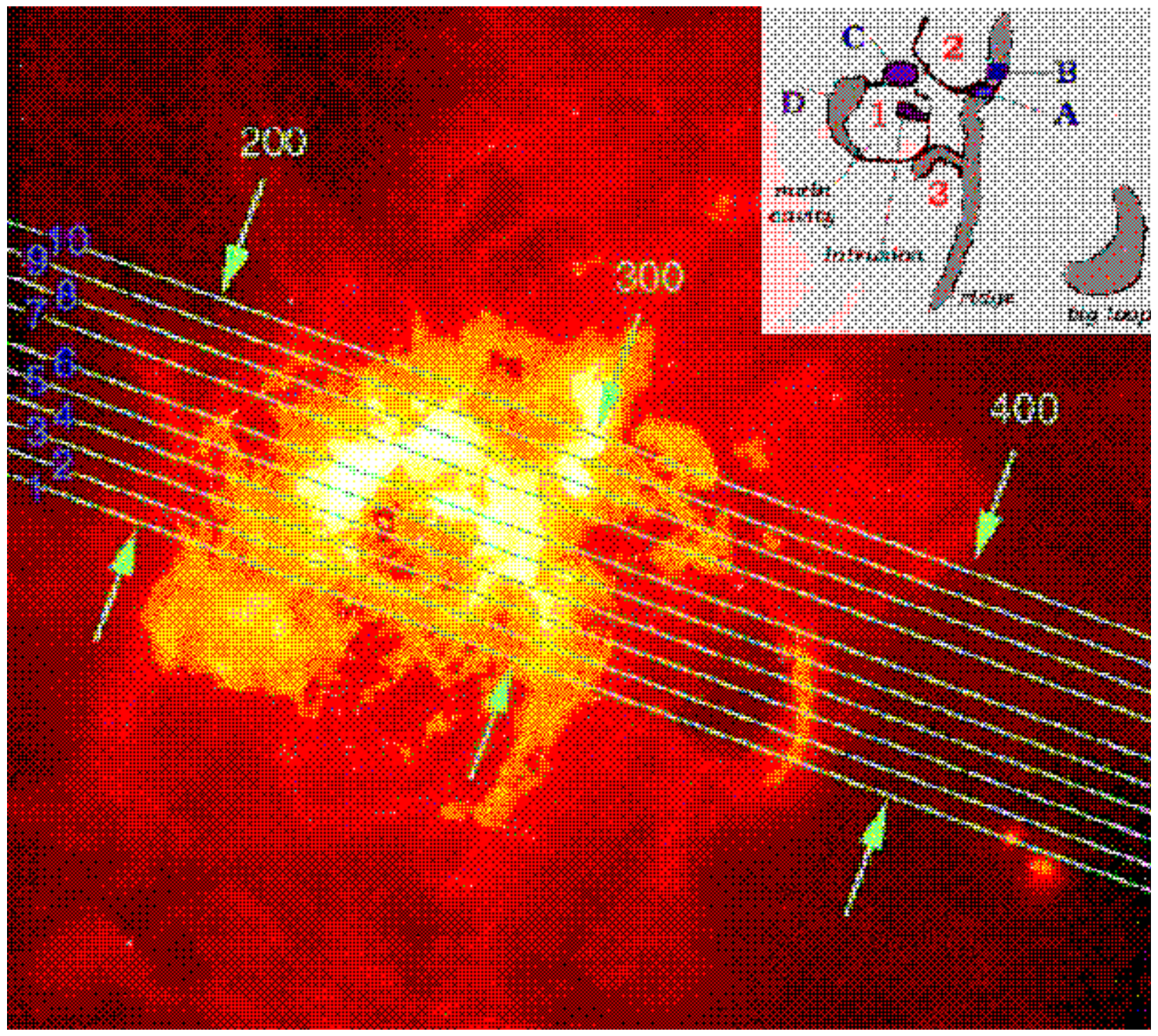

Fig. 2.- NGC 604 HST H $\alpha$ image with overlaid slit locations. The inset sketches the main features discussed in the text. 

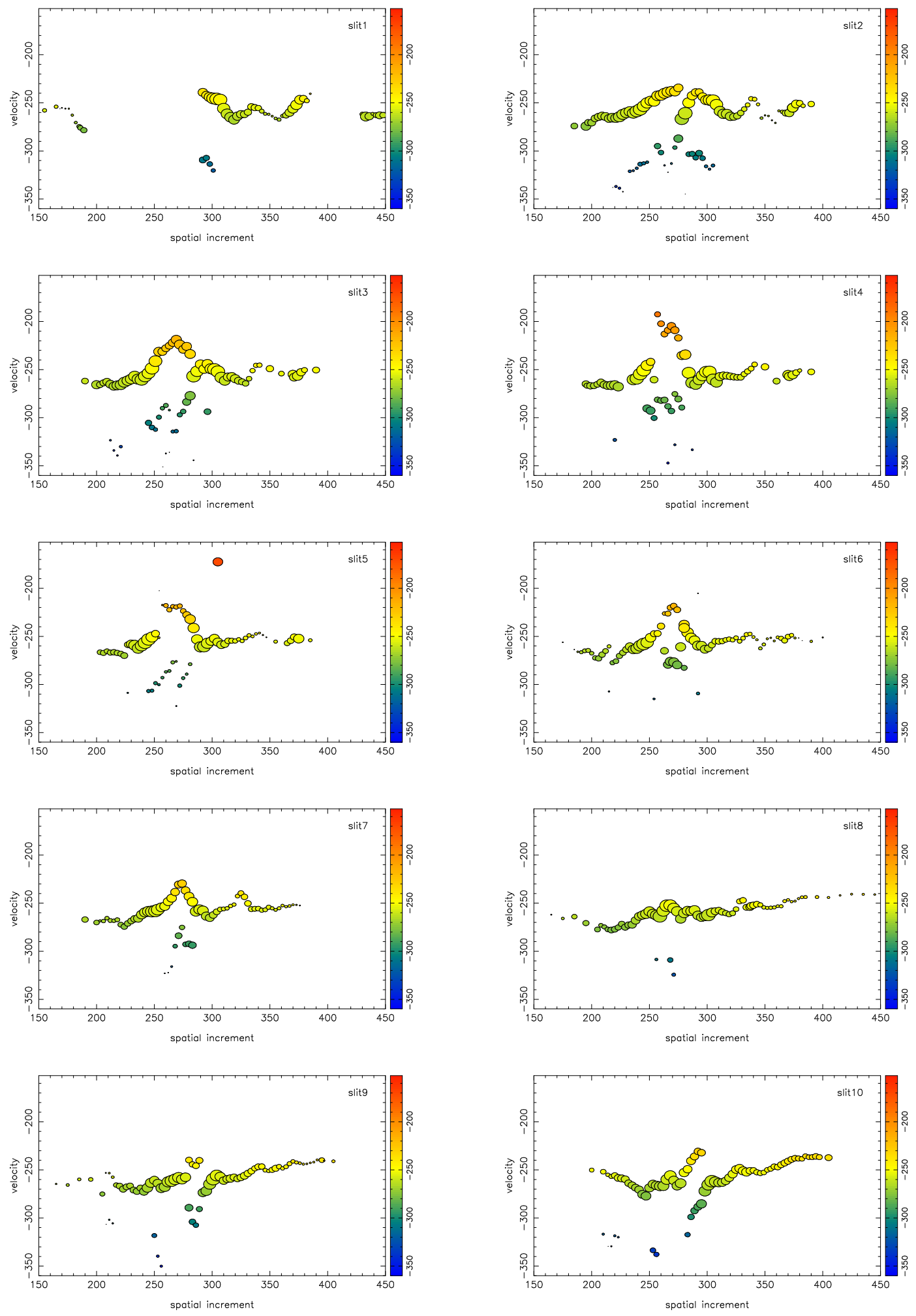

Fig. 3. - $\mathrm{H} \alpha$ velocity along the ten slit positions across the nebula. The size of the symbol is proportional to the $\log$ of the $\mathrm{H} \alpha$ flux within that slit position. The vertical scale is in $\mathrm{km} \mathrm{s}^{-1}$ and the horizontal scale is in long-slit pixels $(1$ pixel $\approx 1.3 \mathrm{pc})$. 

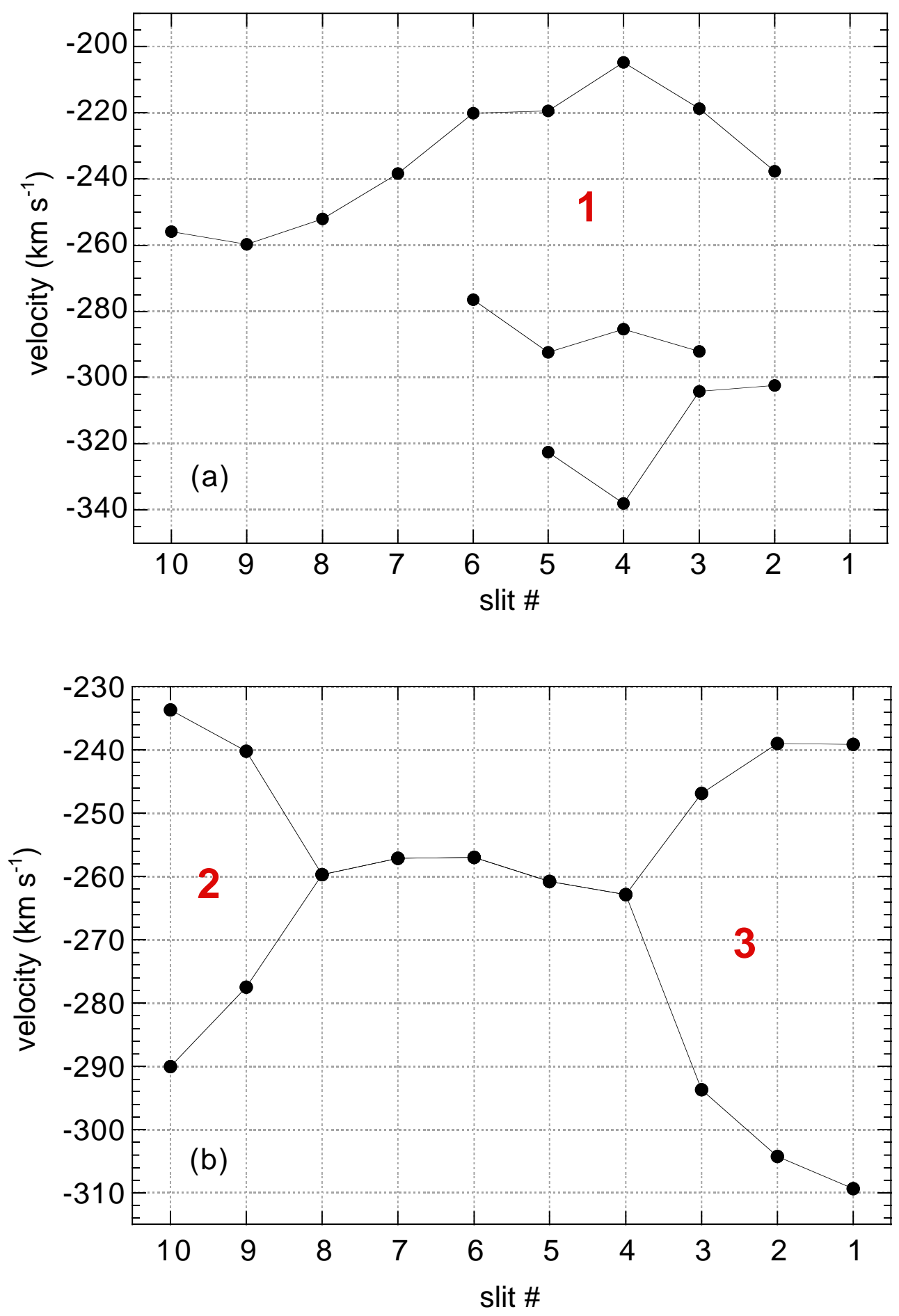

Fig. 4.- The ongoing blowout. Kinematic signatures of the three main disrupted cavities discussed in the text. 